\title{
Quest for dark matter by direct detection experiments with noble liquids
}

\author{
Hiroyuki Sekiya* \\ ICRR and Kavli IPMU, The University of Tokyo \\ E-mail: sekiya@icrr.u-tokyo.ac.jp
}

\begin{abstract}
Recently so many dark matter direct search expriments have been conducted. Among them the detectors with noble liquid gasses, especially liquid Xe detectors, gave the best limit on the dark matter-nucleus cross section so far. In this Proceedings, first, the principle of direct searches and noble liquid technologies are riviewed. Then, as an example, details and recent results of XMASS, the unique single phase liquid Xe scintillator, is described. Finally, future prospects of noble liquid detectors are mentioned.
\end{abstract}

KMI International Symposium 2013 on Quest for the Origin of Particles and the Universe 11-13 December, 2013

Nagoya University, Japan

\footnotetext{
* Speaker.
} 

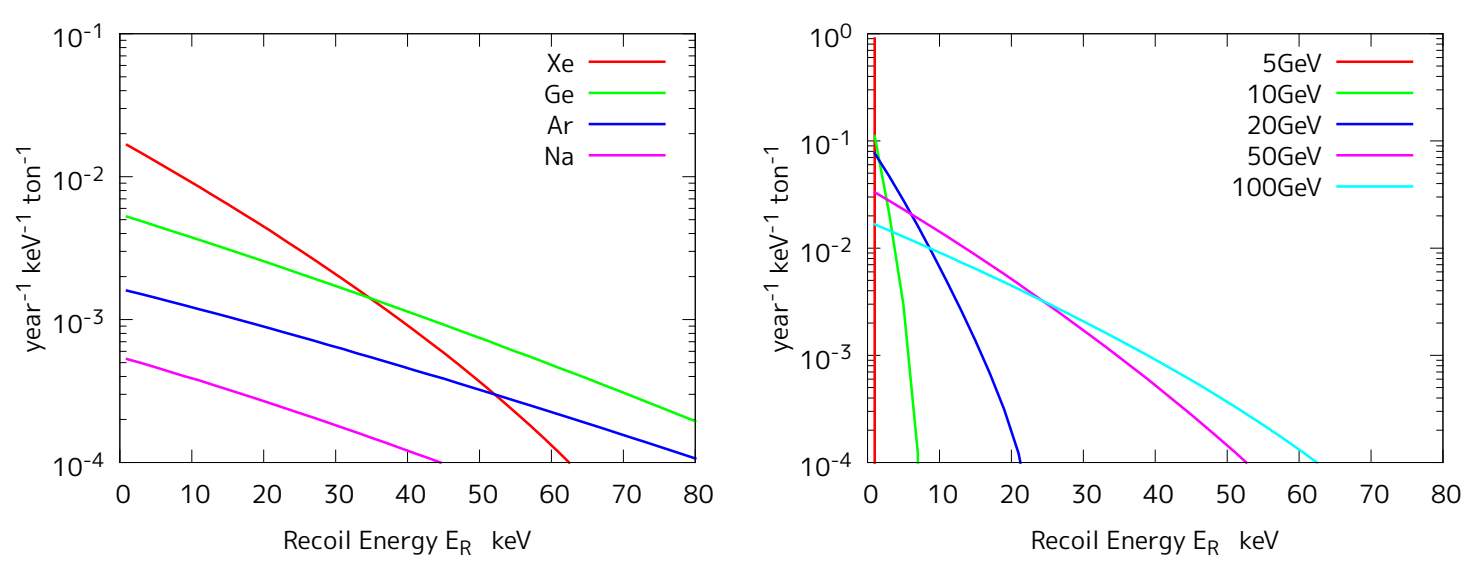

Figure 1: WIMP-induced recoil energy spectra of the spin-independent cross section $\sigma_{\mathrm{WIMP}-\mathrm{n}}^{\mathrm{SI}}=10^{-48} \mathrm{~cm}^{2}$ (Left) for different nuclei in case of $M_{\mathrm{WIMP}}=100 \mathrm{GeV}$ and (Right) for different WIMP mass in case of Xe target.

\section{Direct Dark Matter Detection}

WIMPs are expected to be detected through observation of nuclear recoils produced in their elastic scattering interactions with detector nuclei [1]. The event rate of WIMPs-nuclei scattering depends on the WIMPs flux on earth and the cross section. In principle, the WIMP mass $M_{\text {WIMP }}$ and the interaction cross section can be predicted theoretically based on beyond standard models (BSMs), however, especially after the LHC era, the constraint from the experiments would rather be motivated to discern the "correct BSM" which predicts the WIMP-nucleon cross section $\sigma_{\text {WIMP-n }}$ down to $10^{-48} \mathrm{~cm}^{2}$ [2]. Direct dark matter experiments assume a local halo density and a halo model in order to set an limit on the cross section from the event rates.

The "Standard Halo Model" used so far is the solution of the collision-less Maxwell-Boltzmann equation assuming the halo profile in the Milky Way $\rho(r) \propto r^{-2}$ and the local halo density $\rho_{0}=$ $0.3 \mathrm{GeV} \cdot \mathrm{cm}^{-3}$. The WIMP velocity distribution is Maxwell distribution;

$$
f(\vec{v})=\frac{1}{\pi^{3 / 2} \sigma^{3}} e^{-\frac{|\overrightarrow{\mid}|^{2}}{\sigma^{3}}}
$$

where $\sigma=\sqrt{(3 / 2)} v_{r_{0}}, v_{r_{0}}=220 \mathrm{~km} / \mathrm{s}$ and this distribution is truncated at the escape velocity from the Milky Way, $v_{\text {esc }}=544 \mathrm{~km} / \mathrm{s}$. However recent N-body simulations reveal this assumption is not so realistic. There are indications of deviation from Maxwell distribution, particularly at high velocities [3]. As for the local halo density, it is likely higher than $0.3 \mathrm{GeV} \cdot \mathrm{cm}^{-3}$, e.g. $\rho_{0}=$ $0.39 \mathrm{GeV} \cdot \mathrm{cm}^{-3}$ [4] and $\rho_{0}=0.37 \mathrm{GeV} \cdot \mathrm{cm}^{-3}$ [5]. These deviations from the "Standard Halo Model" have an impact especially on the search for the light WIMPs.

Figure 1 shows the expected WIMP-induced recoil energy spectra of the spin-independent cross section $\sigma_{\mathrm{WIMP}-\mathrm{n}}^{\mathrm{SI}}=10^{-48} \mathrm{~cm}^{2}$ for different nuclei in case of $M_{\mathrm{WIMP}}=100 \mathrm{GeV}$ and for different WIMP mass in case of Xe target. It is clear that heavy nuclei and light WIMPs are easier for experimentalists. 


\section{Direct detection technologies and Noble liquids}

As already discussed, the WIMP nuclear recoil signal rate might be less than 1 events/ton/year and the energy transferred to a nucleus is below $10-100 \mathrm{keV}$. To observe a WIMP induced spectrum, a large target mass, a low energy threshold, and an extremely low background materials are required. In the detectors, the deposit energy is transformed into measurable signals, such as ionization, scintillation, and phonon. The simultaneous detection of more than two signals gives a powerful discrimination ability against most of background events which are electromagnetic recoils. The phonon plus ionization/scintillation techniques are successfully implemented in $[6,7,8]$ detectors.

While liquid noble gasses (noble liquids) such as Ar and Xe offer the simultaneous detection of ionization and scintillation signals. In addition to this feature, noble liquids are excellent media for WIMP search because; (1) In principle, there is no limitation of building large scale homogeneous detectors (2) they have large light yields, and this makes it possible to realize very low threshold detectors (3) even after building the detector, it is possible to purify and lower the background. As a consequence, lots of experiments with noble liquids has been conducted and the current most stringent limits on the cross section for spin-independent couplings are derived from liquid Xe detectors $[9,10,11]$.

According to [12], historically, the ionization signal in noble liquids was studied at first. The high electron mobility in noble liquids was discovered in 1948 [13]. Then, their scintillation properties were studied in 1950's [14] and it was revealed that the scintillation light (S1) of noble liquids is emitted at $178 \mathrm{~nm}$ for liquid Xe and $128 \mathrm{~nm}$ for liquid $\operatorname{Ar}[15,16]$. In the following years, the electron mobility was studied in more detail [17, 18, 19], and many studies focused on ionization; the mean energy to produce an ion-electron pair is $\mathrm{W}=19.5 \mathrm{eV}$ for liquid $\mathrm{Ar}$ and $\mathrm{W}=14.7 \mathrm{eV}$ for liquid Xe [15]. Moreover an electron multiplication was discovered [20] and it was shown that "proportional scintillation"(S2) is present in the both liquid phase and in the gaseous phase [21]. This led to the testing of both direct (S1) and proportional (S2) scintillation in liquid xenon by several groups [22, 23, 24].

Actually there are 3 ways to utilize above features of noble liquids for WIMP searches, i.e. two phase (liquid/gas) TPC, single phase (liquid) TPC, and single phase (liquid) scintillator. The conceptual designs of these 3 detectors are shown in Figure 2.

The most established design so far is the two phase TPC and as already mentioned, several successful implementations gave the best results $[9,10,11]$. The two phase detectors were originally proposed in [25]. The main function of the two phase operation is to measure the direct scintillation (S1) in the liquid and ionization, via proportional scintillation (S2) in the gas. In fact electrons produced by the radiation interaction in the liquid can be accelerated by an electric field in the gas to acquire enough energy to excite molecular states of the gas and produce secondary scintillation. However, this two phase configuration is rather complicated in reality. Three electric fields of three different regions (in liquid, between the liquid surface, and in gas) must be controlled precisely and the liquid level itself also must be adjusted between the grids. These conditions really affect the the performance of the detector with regard to the scintillation response [26], thus making large scale detectors is not easy in terms of stability.

To avoid this complexity, the single phase TPC, the original design of the measuring both 

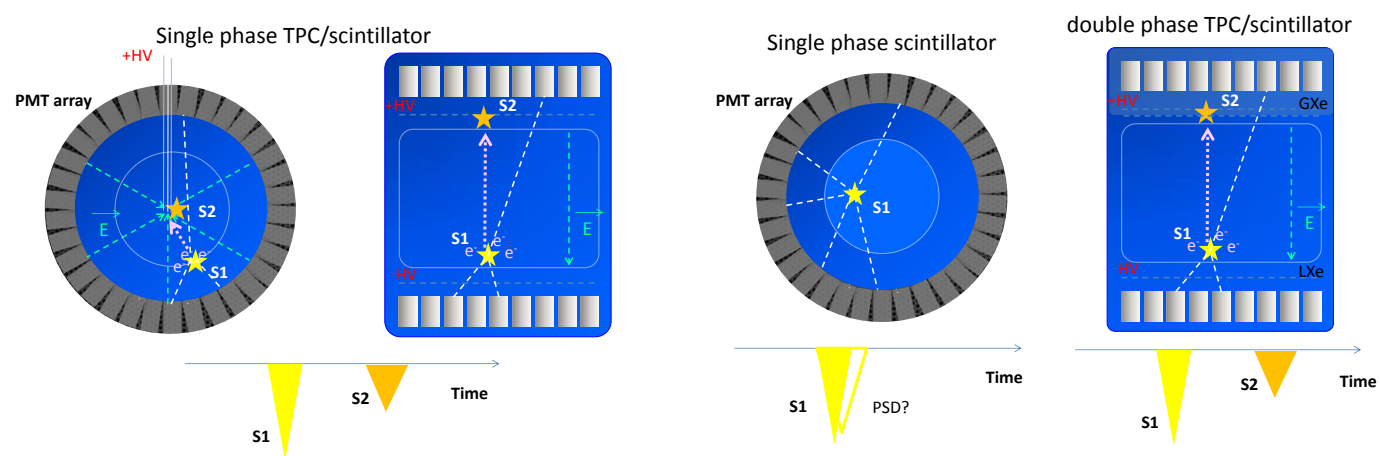

Figure 2: 3 designs of noble liquid WIMP detectors

$\mathrm{S} 1$ and S2 in liquids has been revived recently and are tested [27, 28]. By putting the electron multiplication region into the liquid phase, the management of the fields is somehow relaxed. The electron multiplication can be realized by wires, needles [27], meshes, or micro pattern detectors such as THGEMs [28].

For more simplicity, using noble liquids just as single phase scintillaors was studied in early 1990's [29, 30]. Recently a large ( $\sim 1$ ton) scale Xe detector has become online [31] and an even larger ( $\sim 3.6$ ton) Ar detector is being constructed [32]. Because of the simple design and no electric field, these detectors has an advantage of the large scalability. Besides, although they work just as scintillators, they are also sensitive to the ionization through the recombination process of the ions. Consequently, scintillation timing properties offer the background rejection power by pulse shape discrimination $[32,33]$.

As an example of the implementations of noble liquid, XMASS is described in the next section.

\section{XMASS}

XMASS is a single phase liquid Xe scintillator detector containing $1050 \mathrm{~kg}$ of $\mathrm{Xe}$ in an OFHC copper vessel. As shown in Figure. 3, Xe scintillation light is collected by 630 hexagonal and 12 cylindrical inward-pointing Hamamatsu R10789 series photomultiplier tubes (PMTs) arranged on an $80 \mathrm{~cm}$ diameter pentakis-dodecahedron support structure within the vessel to give a total photocathode coverage of $62.4 \%$. These PMTs view an active target region containing $835 \mathrm{~kg}$ of liquid Xe. In order to monitor the PMT stability and measure the trigger efficiency, eight blue LEDs with Teflon diffusers are mounted to the support structure. There are six LEDs arranged along the equator and one each at the top and the bottom of the pentakis-dodecahedron. To shield the scintillator volume from external gammas, neutrons, and muon-induced backgrounds, the copper vessel is placed at the center of a $\phi 10 \mathrm{~m} \times 11 \mathrm{~m}$ cylindrical tank filled with pure water. This volume is viewed by 72 Hamamatsu R3600 20-inch PMTs to provide both an active muon veto and passive shielding against these backgrounds. This is the first water Cherenkov shield used in a dark matter search experiment. To perform energy and position reconstruction calibrations a portal has been prepared along the central vertical axis (z-axis) of the PMT support structure through which an 


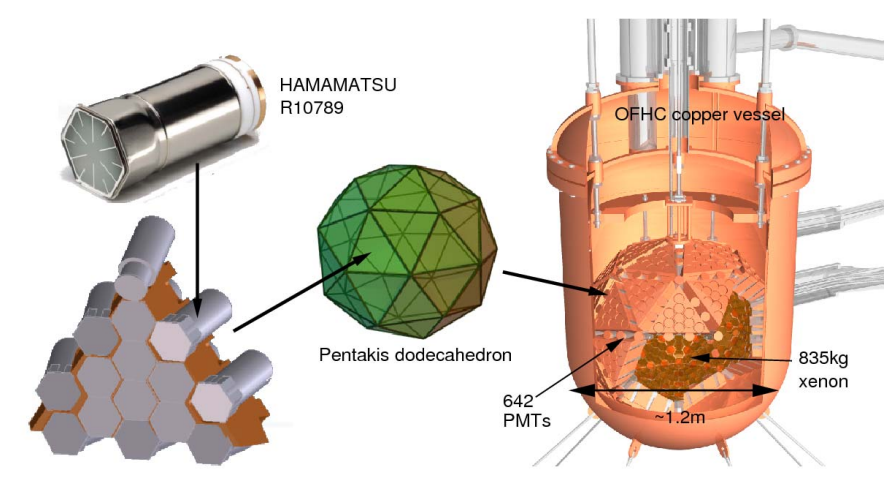

Figure 3: Structure of the XMASS detector.

OFHC copper rod can be inserted directly into the target volume. Thin cylindrical calibration sources containing either of ${ }^{55} \mathrm{Fe},{ }^{57} \mathrm{Co},{ }^{109} \mathrm{Cd}$, or ${ }^{241} \mathrm{Am}$ are placed at the tip of this rod to perform detector calibrations. A more detailed description of the XMASS detector is presented in [31].

The scientific data, corresponding to 6.70 days of livetime, was taken in February 2012 with a low trigger threshold of four PMT hits [34]. Using $122 \mathrm{keV}$ gammas from the ${ }^{57} \mathrm{Co}$ calibration source the Xe light yield was found to be 14.7 photoelectrons/keVee. This large light yield allows the analysis threshold to be lowered sufficiently for sensitivity to low mass WIMPs. In order to achieve optimal sensitivity, the entire detector volume is used because fiducialization is increasingly difficult at these low energies. A sequence of data reduction is applied to remove events caused by the tail of the scintillation light distribution after energetic events; (1) events triggered only with the liquid Xe detector are selected, (2) events that occurred within $10 \mathrm{~ms}$ of the previous event are rejected, and (3) events whose timing distribution has an RMS greater than $100 \mathrm{~ns}$ are removed. The last cut is applied to remove Cherenkov events originated from ${ }^{40} \mathrm{~K}$ contamination in the PMT photocathodes; events with more than $60 \%$ of their PMT hits occurring within the first $20 \mathrm{~ns}$ of the event window are removed as Cherenkov-like.

Figure 4 shows simulated WIMPs energy spectra overlaid on the observed spectrum after the data reduction was applied. In order to set a conservative upper bound on the spin-independent WIMP-nucleon cross section, the cross section is adjusted until the expected event rate in XMASS does not exceed the observed one in any energy bin above the analysis threshold. It is chosen as the energy at which the trigger efficiency is greater than $50 \%$ for $5 \mathrm{GeV}$ WIMPs and corresponds to 0.3 keVee. The resulting $90 \%$ confidence level (C.L.) limit is also shown in Figure 4.

After careful study of the events surviving the analysis cuts, their origins are not completely understood. Contamination of ${ }^{14} \mathrm{C}$ in the GORE-TEX ${ }^{\circledR}$ sheets between the PMTs and the support structure may explain a fraction of the events. Light leaks through this material are also suspect. Nonetheless, the possible existence of a WIMP signal hidden under these and other backgrounds cannot be excluded. Although no discrimination has been made between nuclear-recoil and electronic events, and many events remain in the analysis sample, the present result excludes part of the parameter space favored by other measurements $[7,35,36]$ when those data are interpreted as a signal for light mass WIMPs.

Lastly, we have modified the inner surface of XMASS, especially around the PMTs, to improve 

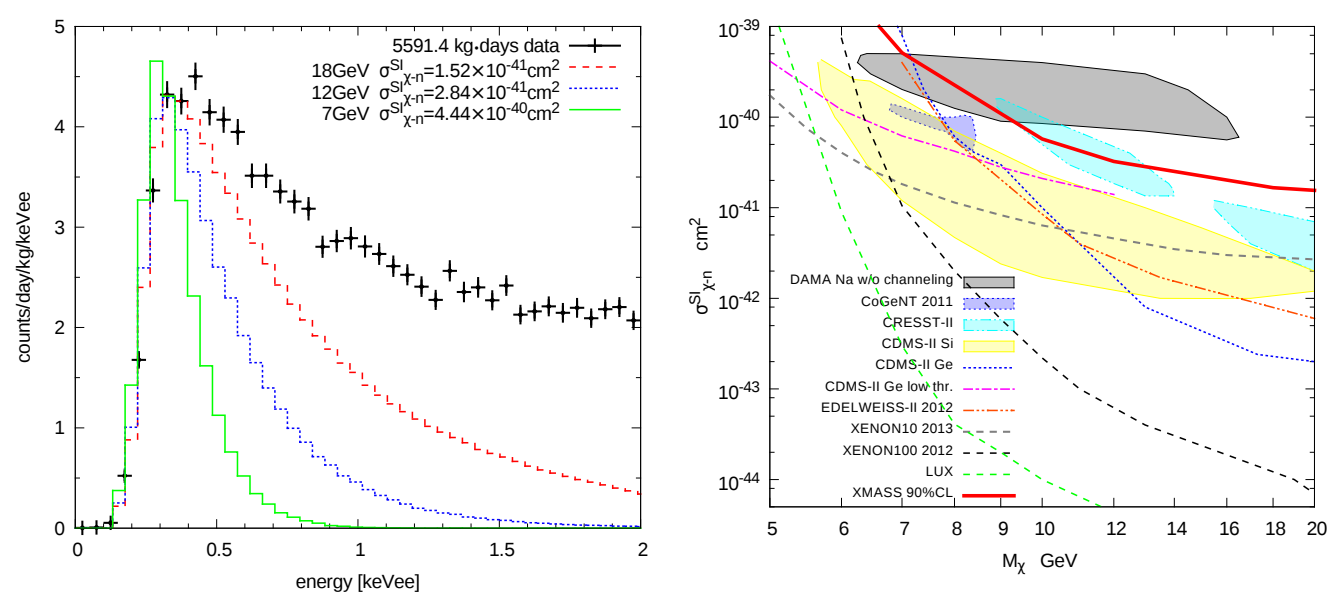

Figure 4: (Left)Simulated WIMP energy spectra in the XMASS detector assuming the maximum cross section that provides a signal rate no larger than the observation in any bin above $0.3 \mathrm{keVee}$. (Right)Spinindependent elastic WIMP-nucleon cross section as a function of WIMP mass. Limits from other experiments and favored regions are also shown $[6,7,8,9,10,35,36,37]$.

the detector performance, and have resumed scientific data acquisition.

\section{Future prospects of noble liquid detectors}

Since we have no convincing evidence of a direct detection signal by WIMPs so far, it has become clear that, at least ton-scale experiments are indispensable for a discovery. It is encouraging that several multi-ton scale noble liquid detectors are planned or already in the construction phase [32, 38, 39, 40, 41]. In further future, very huge two phase detectors, using both $\mathrm{Ar}$ and $\mathrm{Xe}$ are under study. MAX is the US project, with $70 \mathrm{t}$ and $20 \mathrm{t}$ total Ar and Xe detectors, respectively. [42] DARWIN is an European proposal for $20 \mathrm{t} \mathrm{Xe} \mathrm{and/or} \mathrm{Ar} \mathrm{TPCs} \mathrm{[43].} \mathrm{In} \mathrm{the} \mathrm{single} \mathrm{phase}$ detector side, XMASS-II with 24t Xe is proposed in Japan [44].

These detectors also aim to extend physics programs such as the detection of low-energy solar neutrinos and of the neutrino-less double beta decay in ${ }^{136} \mathrm{Xe}$. Actually, their sensitivity to WIMPnuclei interactions will be limited by the pp-solar neutrino flux, which gives a lower limit on the attainable cross section of $\sim 10^{48} \mathrm{~cm}^{2}$ [44].

Finally, next generation of WIMP direct detection experiments are expected to have the potential to go beyond the discovery and reveal detailed information about both the particle physics and astrophysics.

\section{References}

[1] M.W. Goodman and E. Witten, Phys. Rev. D31, (1985) 2059.

[2] J. Ellis, arXiv:1312.5426.

[3] Y.Y. Mao, L.E. Strigari, R.H. Wechsler, H.Y Wu, and O. Hahn, J. of Cosmo. and Astropart. Phys. 02 (2010) 030. 
[4] R. Catena and P. Ullio, arXiv:0907.0018v2.

[5] F.S. Ling, E. Nezri, E. Athanassoul and R. Teyssier, J. of Cosmo. and Astropart. Phys. 02 (2010) 012.

[6] Z. Ahmed, et al., Science, 327 (2010), 1619; Z. Ahmed, et al., Phys. Rev. Lett. 106 (2011) 131302; R. Agnese, et al., Phys. Rev. Lett. (2013), arXiv:1304.4279.

[7] G. Angloher, et al., Eur. Phys. J., C72 (2012) 1971.

[8] E. Armengaud, et al., Phys. Lett., B702 (2011) 329.

[9] D.S. Akerib, et al., arXiv:1310.8214.

[10] E. April, et al., Phys. Rev. Lett. 109, (2012) 181301.

[11] D. Akimov, et al., Phys. Lett. 709, (2012) 14.

[12] R. Bernabei, P. Belli, A. Incicchitti and D Prosperi, arXiv:0806.0011v2

[13] N. Davidson and A.E. Larsh, Phys. Rev. 74 (1948) 220; G.W. Hutchinson, Nature 162 (1948) 610.

[14] J.A. Nortrop and J.M. Gursky, Nucl. Instr. and Meth. 3 (1958) 207; J.A. Nortrop, J.M. Gursky and A.E. Johnsrund, IEEE Trans. Nucl. Sci. NS-5 (1958) 81.

[15] T. Doke et al., Jpn. J. Appl. Phys. 41 (2002) 1538.

[16] R.E. Packard, F. Reif and C.M. Surko, Phys. Rev. Lett. 25 (1970) 1435.

[17] J.H. Marshall, Rev. Sci. Instr. 25 (1954) 232.

[18] L.W. Alvarez, LRLP Note n. 672 (1968).

[19] T. Doke, Butsuri (Monthly Rev. Journal published in Japanese by the Phys. Soc. of Japan) 24 (1969) 609.

[20] R.A. Muller et al., Phys. Rev. Lett. 27 (1971) 532.

[21] A. Lansiart et al., Nucl. Instr. and Meth. 135 (1976) 47.

[22] M. Miyajima et al., Nucl. Instr. and Meth. 160 (1979) 239; K. Masuda et al., Nucl. Instr. and Meth. 160 (1979) 247.

[23] A.J.P.L. Policarpo, Nucl. Instr. and Meth.196 (1982) 53; A.J.P.L. Policarpo et al., Nucl. Instr. and Meth. A 365 (1995) 568.

[24] P. Benetti et al., Nucl. Instr. and Meth. A 327 (1993) 203.

[25] B.A. Dolgoshein, V.N. Lebedenko and B.U. Rodionov, JETP Lett. 11 (1970) 513.

[26] F. Favata et al., Nucl. Instr. and Meth. A 294 (1990) 595.

[27] H. Sekiya, Proceedings of the 6th International Symposium on large TPCs for Low Energy Rare Event Detection (2012); arXiv:1301.7125.

[28] L. Arazi et al., Proceedings of the 3rd International Conference on Micro Pattern Gaseous Detectors (2013); arXiv:1310.4074.

[29] P. Belli, et al., N. Cim. A 103(1990) 767.

[30] G.J. Davies, et al., Phys. Lett. B 320 (1994) 395.

[31] K. Abe, et al., Nucl. Instr. and Meth. A 716 (2013) 78. 
[32] M.G. Boulay, et al., Proceedings of the 12th International Conference on Topics in Astroparticle and Underground Physics (2011); arXiv:1203.0604.

[33] K Ueshima, et al., Nucl. Instr. and Meth. A 595 (2008) 148.

[34] K. Abe, et al., Phys. Lett. B 719 (2013) 78.

[35] R. Bernabei, et al., Eur. Phys. J. C 56 (2008) 333; R. Bernabei, et al., Eur. Phys. J. C 67 (2010) 39.

[36] C.E. Aalseth, et al., Phys. Rev. Lett. 106 (2011) 131301; Phys. Rev. D 88 (2013) 012002.

[37] J. Angle, et al., Phys. Rev. Lett. 107 (2011) 051301.

[38] E. April, et al., arXiv:1206.6288.

[39] A. Wright, et al., arXiv:1109.2979.

[40] H. Nelson, et al., Dark Matter 2014 conference.

[41] S. Moriyama, et al., Dark Matter 2014 conference.

[42] R. Alarcon, S. Balascuta., D. Alton, E. Aprile, K.L. Giboni et al., FERMILABPROPOSAL-1001 (2009) 24.

[43] L. Baudis et al., J. Phys. Conf. Ser. 375 (2012) 012028.

[44] Y. Suzuki, et al., arXiv:hep-ph/0008296. 